\title{
Prácticas de riesgo para transmisión de VIH en estudiantes de medicina de Latinoamérica y el Caribe, 2017
}

\author{
*Carlos Miguel Rios-González ${ }^{1}$, Fabiana Griselda Verón-Mellid ${ }^{2}$, Ginno Alessandro De Benedictis- \\ Serrano $^{3}$, Jessica F. Flores-Enríquez ${ }^{4}$, Alfonzo David Chirino-Caicedo ${ }^{3}$
}

${ }^{1}$ Universidad Nihon Gakko, Dirección de Investigación. Fernando de la Mora, Paraguay

2Universidad Nacional de Caaguazú, Facultad de Ciencias Médicas. Cnel. Oviedo, Paraguay

${ }^{3}$ Universidad de Carabobo Sede Aragua, Facultad de Ciencias de la Salud. Venezuela

${ }^{4}$ Universidad Central del Ecuador, Facultad de Ciencias Médicas. Ecuador

Cómo referenciar este artículo/ How to reference this article:

\author{
Rios-González CM, Verón-Mellid FG, De \\ Benedictis-Serrano GA, Flores-Enríquez JF, \\ Chirino-Caicedo AD. Prácticas de riesgo para \\ transmisión de VIH en estudiantes de medicina de \\ Latinoamérica y el Caribe, 2017. Mem. Inst. Investig. \\ Cienc. Salud. 2018; 16(2): 49-54
}

\section{R E S U M E N}

El objetivo del trabajo fue determinar las prácticas de riesgo para transmisión de VIH en estudiantes de medicina de Latinoamérica y el Caribe durante los meses de Enero a Febrero de 2017.

Se realizó un estudio observacional, descriptivo de corte transversal, con muestreo no probabilístico por conveniencia en estudiantes de medicina de Latinoamérica y el Caribe, desde enero a febrero de 2017. Se escogió a estudiantes de medicina del primer al séptimo año de la carrera. Se procedió a la publicación en redes sociales del cuestionario electrónico mediante Google Doc(C). Los resultados se expresan en tablas de frecuencias. Fueron incluidos 638 estudiantes de medicina, teniendo el 67,08\% (428) una edad comprendida entre 20 a 24 años. Del total de encuestados el 56,02\% ha practicado sexo anal, 88,65\% ha practicado sexo oral, y 57,06\% sexo vaginal en los últimos 6 meses. El 24,43\% de los encuestados nunca ha utilizado condón durante sus relaciones sexuales. El 3,49\% han pagado alguna vez por sexo, $27,57 \%$ ha ingerido alcohol durante las relaciones sexuales y $4,36 \%$ ha mantenido relaciones sexuales con usuarios de drogas intravenosas mientras que $63,88 \%$ nunca se ha hecho una prueba rápida para VIH. Las prácticas de riesgos más frecuentes fueron la baja utilización de condón durante la última relación sexual, la utilización de alcohol durante la relación sexual y la baja realización de prueba para VIH.

Palabras clave: VIH, gestión de riesgos, estudiantes de medicina.

\section{Risk practices for HIV transmission among medical students in Latin America and the Caribbean, 2017}

\section{A B S T R A C T}

The objective was to determine the risk practices for HIV transmission among medical students from Latin America and the Caribbean from January to February 2017. This was an observational descriptive cross-sectional study with non-probabilistic sampling for convenience in medical students from Latin America and the Caribbean that was carried out from January to February 2017. Medical students were selected from the first to the seventh year of the career. The electronic questionnaire was published in social networks using Google Doc (c). The data were expressed in tables of frequencies. Six hundred and thirty eight medical students were included, 67.08\% (428) with ages between 20 and 24 years. Of the total number of respondents, $56.02 \%$ had anal sex, $88.65 \%$ had oral sex, and $57.06 \%$ had vaginal sex in the last 6 months. The $24.43 \%$ of the respondents have never used a condom during their sexual relations, $3.49 \%$ have paid for sex, $27.57 \%$ drank alcohol during sex, and $4.36 \%$ have had sex with intravenous drug users while $63.88 \%$ 
have never had a quick test for HIV. The most frequent risk practices were the low condom use during the last sexual intercourse, the use of alcohol during intercourse and the low HIV test performance.

Keywords: HIV, risk management, medical students.

\section{INTRODUCCIÓN}

La infección por el VIH, sigue constituyendo un problema de salud pública, debido a la amplia distribución en todo el mundo y al estigma social que acarrea(1). Según la Organización Mundial de la Salud (OMS), en el 2015 se produjeron $2[1,8-2,4]$ millones de nuevas infecciones por VIH, a su vez se estima que unos 35 millones de personas han fallecido por causas relacionadas con el SIDA hasta ese mismo año(2).

Entre las poblaciones más vulnerables se encuentran los jóvenes de entre 15 y 24 años que representan el $45 \%$ estimado del total de las nuevas infecciones por año en todo el mundo, siendo su principal vía de transmisión la sexual, con una frecuencia relativa de $98 \%$ de los $\operatorname{casos}^{(3,4)}$. Otro grupo de riesgo son las poblaciones indígenas, las embarazadas, usuarios de drogas intravenosas, y los trabajadores sexuales, entre otros ${ }^{(5)}$. Se identifican como prácticas de riesgo, las prácticas sexuales (anal, vaginal u oral) sin preservativo, consumo de drogas tales como el alcohol, marihuana durante las relaciones sexuales, así como el uso de drogas intravenosas entre otros ${ }^{(6,7)}$.

Se asume que el mayor conocimiento que presumiblemente tienen los estudiantes de medicina en áreas del cuidado de la salud representa un factor protector en el desarrollo de actitudes y comportamientos saludables en salud sexual y reproductiva ${ }^{(8)}$, sin embargo, de acuerdo a estudios previos, el buen nivel de conocimientos no es suficiente para evitar conductas de riesgo por lo que es de suma importancia el estudio de los estudiantes de medicina, debido a que su profesión produce un impacto en la sociedad, ya que es un actor directamente relacionado al autocuidado y los hábitos saludables de sus pacientes y entorno ${ }^{(9)}$.

Con base en todo lo anterior nos planteamos como objetivo determinar las prácticas de riesgo para transmisión de VIH en estudiantes de medicina de Latinoamérica y el Caribe durante enero a febrero de 2017.

\section{MATERIAL Y MÉTODOS}

Estudio observacional, descriptivo de corte transversal, con muestreo por conveniencia en estudiantes de medicina de Latinoamérica y el Caribe del primer al octavo año de la carrera (en Honduras la carrera dura ocho años), entre los meses de enero a febrero de 2017 que hayan accedido a llenar el cuestionario. Los estudiantes de medicina eran de países miembros de la Federación Latinoamericana de Estudiantes de Medicina (FELSOCEM). La encuesta utilizada fue anónima y autoadministrada. Respecto a los aspectos éticos, se informó a los participantes sobre los objetivos del estudio y se solicitó la autorización para el uso de sus datos en este estudio firmando un consentimiento informado. Tuvo la aprobación del Comité de Ética de la Universidad Central de Ecuador. Toda la información de las encuestas fue confidencial. Por parte de los investigadores no se divulgó, repitió o comentó la información proporcionadas por las fichas.

Para la recolección de la muestra se utilizó el Cuestionario sobre riesgo de enfermedades de transmisión sexual/Virus de Inmunodeficiencia Humana/ Hepatitis diseñado y validado por el Departamento de Salud del Estado de Nueva York, Oficina de Enfermedades Contagiosas, validado por expertos para la aplicación en este estudio(10,11). El cuestionario estuvo dividido en 3 secciones, la primera de las variables epidemiológicas, la segunda de las variables sobre las prácticas sexuales, y la tercera sobre las prácticas de riesgo. El cuestionario fue divulgado a través de las redes sociales (Facebook, Instagram, Twitter), mensajería instantánea (WhatsApp), y correo electrónico del cuestionario electrónico mediante Google Doc(C). A todos los participantes del estudio se les notificó mediante una carta de los objetivos del estudio y aquellos que estuvieron de acuerdo, procedieron a Ilenarlo.

Para estimar el tamaño de la muestra se utilizó el paquete epidemiológico EpiInfo $7.0^{\mathrm{TM}}$, para un tamaño poblacional infinito, una proporción esperada de $50 \%$, una precisión de $5 \%$, un nivel de confianza de 95\%, el tamaño muestral mínimo fue de 384 . 
Los datos fueron ingresados a una hoja de cálculo de Microsoft Office Excel(C) 2016, luego del control de calidad de los datos, las variables fueron exportadas y analizadas con el software estadístico Stata $14.0 \AA$. Los resultados se presentaron en medidas de tendencia central, dispersión y tabla de proporciones.

\section{RESULTADOS}

Fueron encuestados 638 estudiantes de medicina entre enero a febrero del 2017, de los cuales el $67,08 \%$ (428) pertenecía al rango etario de 20 a 24 años, el 59,72\% (381) era del sexo femenino, el $24,29 \%$ (155) se encontraban cursando el cuarto año. El $75,54 \%$ de los encuestados afirma haber tenido relaciones sexuales, según la orientación sexual el 38,91\% es heterosexual, 35,60\% homosexual y 25,47 bisexual. La mayor participación de los encuestados fueron de Colombia con un $21,63 \%$ y Perú con $18,81 \%$ (Tabla 1 ).

Tabla 1: Distribución de acuerdo a características generales de los estudiantes de medicina de Latinoamérica, 2017

\begin{tabular}{|c|c|c|}
\hline Características generales & $\mathbf{n}$ & $\%$ \\
\hline \multicolumn{3}{|l|}{ Franja etaria( años) } \\
\hline 18 a 19 & 148 & 23.20 \\
\hline 20 a 24 & 428 & 67.08 \\
\hline 25 a 29 & 56 & 8.78 \\
\hline$\geq 30$ & 6 & 0.94 \\
\hline \multicolumn{3}{|l|}{ Sexo } \\
\hline Masculino & 257 & 40,28 \\
\hline Femenino & 381 & 59,72 \\
\hline \multicolumn{3}{|c|}{ Año de estudio (cursando actualmente) } \\
\hline Primero & 65 & 10.19 \\
\hline Segundo & 112 & 17.55 \\
\hline Tercero & 102 & 15.99 \\
\hline Cuarto & 155 & 24.29 \\
\hline Quinto & 131 & 20.53 \\
\hline Sexto & 58 & 9.09 \\
\hline Séptimo & 8 & 1.25 \\
\hline Octavo & 7 & 1.10 \\
\hline \multicolumn{3}{|l|}{ País } \\
\hline Colombia & 138 & 21.63 \\
\hline Perú & 120 & 18,81 \\
\hline Ecuador & 108 & 16.93 \\
\hline Venezuela & 99 & 15.52 \\
\hline Paraguay & 95 & 14.89 \\
\hline Bolivia & 18 & 2.82 \\
\hline Panamá & 17 & 2,66 \\
\hline Argentina & 16 & 2.51 \\
\hline Honduras & 14 & 2.19 \\
\hline Costa Rica & 9 & 1.41 \\
\hline México & 2 & 0,31 \\
\hline Otro (Guatemala, Uruguay) & 2 & 0.31 \\
\hline \multicolumn{3}{|l|}{ Relaciones sexuales } \\
\hline Si & 573 & 89,67 \\
\hline No & 66 & 10,32 \\
\hline \multicolumn{3}{|l|}{ Orientación sexual } \\
\hline Heterosexual & 223 & 38,91 \\
\hline Homosexual & 204 & 35,60 \\
\hline Bisexual & 146 & 25,47 \\
\hline
\end{tabular}

Del total de encuestados el $56,02 \%$ ha practicado sexo anal, $88,65 \%$ ha practicado sexo oral, y 57,06\% sexo vaginal en los últimos 6 meses. El 3,49\% han pagado alguna vez por sexo, $27,57 \%$ ha involucrado alguna vez alcohol durante las relaciones sexuales y $4,36 \%$ ha mantenido relaciones sexuales con usuarios de drogas intravenosa. El $75,39 \%$ ha consumido alguna vez marihuana, $5,58 \%$ de los encuestados se ha inyectado alguna vez droga intravenosa. $18,67 \%$ afirma haber recibido transfusiones sanguíneas alguna vez. El $43,45 \%$ del total de encuestados tiene tatuajes, 56,02\% tiene perforaciones aparte del de la oreja. El 63,88\% nunca se ha hecho una prueba rápida para VIH. (Tabla 2). 
Tabla 2: Prácticas sexuales o prácticas de riesgo de los estudiantes de medicina de Latinoamérica, 2017

\begin{tabular}{|c|c|c|c|}
\hline Prácticas & $\begin{array}{l}\text { Si } \\
\text { n (\%) }\end{array}$ & $\begin{array}{l}\text { No } \\
\text { n (\%) }\end{array}$ & $\begin{array}{l}\text { No sabe } \\
\text { n (\%) }\end{array}$ \\
\hline \multicolumn{4}{|l|}{ Prácticas sexuales } \\
\hline Practica sexo anal & $321(56,02)$ & $252(43,98)$ & \\
\hline Practica sexo oral & $508(88,65)$ & $65(11,35)$ & \\
\hline Practica sexo vaginal & $327(57,06)$ & $248(42,94)$ & \\
\hline Pago por relaciones sexuales & $20(3,49)$ & $553(96,50)$ & \\
\hline Alcohol durante relaciones sexuales & $158(27,57)$ & $415(72,45)$ & \\
\hline $\begin{array}{l}\text { Mantuvo relaciones sexuales con usuario de droga IV } \\
\text { Prácticas de riesgo }\end{array}$ & $25(4,36)$ & $149(25,47)$ & $399(69,63)$ \\
\hline Alguna vez consumió marihuana & $432(75,39)$ & $141(24,60)$ & \\
\hline Alguna vez se ha inyectado droga IV & $32(5,58)$ & $541(94,41)$ & \\
\hline Alguna vez ha aspirado drogas & $105(18,32)$ & $468(81,67)$ & \\
\hline Alguna vez ha ido a la cárcel & $9(1,57)$ & $564(98,42)$ & \\
\hline Alguna vez le han recibido transfusiones sanguínea & $107(18,67)$ & $201(35,07)$ & $265(46,24)$ \\
\hline Tiene tatuajes & $249(43,45)$ & $324(56,54)$ & \\
\hline Tiene perforaciones (aparte de la oreja) & $321(56,02)$ & $252(43,98)$ & \\
\hline Alguna vez le hicieron un análisis de VIH & $207(36,12)$ & $366(63,88)$ & \\
\hline
\end{tabular}

El $24,43 \%$ de los encuestados nunca ha utilizado condón durante sus relaciones sexuales. Tabla 3

Tabla 3. Uso de condón durante sus relaciones sexuales de los estudiantes de medicina de Latinoamérica, 2017

\begin{tabular}{ll}
\hline Uso de condón & n (\%) \\
\hline Siempre & $40(6,98)$ \\
La mayoría de la veces & $156(27,22)$ \\
No muy frecuentemente & $237(41,36)$ \\
Nunca & $140(24,43)$ \\
\hline
\end{tabular}

\section{DISCUSIÓN}

Las personas que viven con el virus del sida (PVVS) que son conscientes de su estado seropositivo pueden seguir participando en comportamientos de salud de alto riesgo (HRB, por sus siglas en inglés), las prácticas sexuales inseguras (PSU) y el uso de drogas intravenosas entre las PVVIH representan importantes fuentes de transmisión de enfermedades secundarias ${ }^{(12)}$.

En la actualidad se habla de una pandemia de VIH alrededor del mundo siendo uno de los principales medios de transmisión las prácticas sexuales inadecuadas, donde la población de riesgo en aumento son los menores de 25 años, que por lo general es la universitaria $^{(11,12)}$.

El estudio de las poblaciones universitarias como población de riesgo para la transmisión de VIH se relaciona con el conocimiento que deberían tener en este nivel educativo y las prácticas de riesgo que pueden realizar a diario ${ }^{(13-15)}$. Se ha demostrado que el área de estudio universitario tiene una relación directa con los conocimientos y los comportamientos que tenga el estudiante con respecto al VIH y sus prácticas de riesgo, si bien existen estudios que indican que existe un mayor riesgo en estudiantes universitarios del área de Humanidades en comparación con los estudiantes del área de Salud, sigue siendo imperativo el estudio de estas poblaciones en su contexto sociocultural ${ }^{(16,17)}$.

La gran mayoría afirma ser sexualmente activos, en donde la orientación sexual referida fue muy equilibrada entre heterosexualidad, homosexualidad y bisexualidad. Es común que las prácticas de riesgo se asocien a la población no heterosexual, sin embargo, esta carga más bien es estigmática y en un error en el juzgamiento $\operatorname{vulgar}^{(18)}$. En la actualidad la sexualidad femenina ya no es un tabú, por ende se observa una mayor libertad de las mujeres con respecto a las relaciones sexuales, lo cual se evidencia en el estudio ya que el $54,35 \%$ de los estudiantes han tenido relaciones sexuales son mujeres ${ }^{(19)}$. 
De la muestra estudiada el $41,36 \%$ usa de manera poco frecuente el condón en sus relaciones sexuales, pero es de alarmar que el $24,43 \%$ refiere no usar condón en sus prácticas sexuales. Esto puede ser debido al uso actual de anticonceptivos orales y otros métodos de anticoncepción, pero aun siendo estudiantes de Medicina descuidan el riesgo de transmisión de VIH y otras enfermedades de transmisión sexual ${ }^{(20,21)}$.

El $27.57 \%$ de la población ha involucrado alcohol durante sus relaciones sexuales, lo cual se puede entender como una actividad correspondiente a la edad, sin embargo, esto puede llevar a facilitar el riesgo de transmisión de $\mathrm{VIH}^{(22)}$. Muchas de las prácticas que realizan los estudiantes no son totalmente seguras, ya que el $75,39 \%$ ha consumido marihuana lo cual es una conducta adoptada por los jóvenes actualmente ${ }^{(23)}$, el $43.45 \%$ tiene tatuajes y un $56,02 \%$ tiene perforaciones además de las de las orejas, lo que lleva a entender que un alto porcentaje de los estudiantes se mantiene realizando actividades de riesgo que pueden llevar a la primo-infección de $\mathrm{VIH}^{(24)}$.

La transfusión sanguínea es un riesgo debido a la probabilidad de adquirir VIH u otras enfermedades, sin embargo, existen situaciones que ameritan este procedimiento como en el caso de los encuestados en donde el 18,67\% afirma haber recibido alguna transfusión sanguínea.

Posterior a todo lo discutido es alarmante que el $63,88 \%$ nunca se ha hecho una prueba de análisis para VIH, lo cual puede indicar que alguno de los encuestados pueda encontrarse en periodo de latencia y no saberlo, esto puede llevar a que nuestro estudio sea un aviso de la situación de riesgo a la que se mantienen expuestos los estudiantes de medicina que se supone tienen los conocimientos de prevención debido a su área de estudio.

Las limitaciones del estudio se centran en que la muestra provino de un muestreo no probabilístico, por lo que no se puede generalizar los resultados, en vista de lo cual consideramos necesario conducir nuevos estudios que puedan ampliar las variables discutidas, ya que es impactante que estudiantes de medicina demuestren prácticas de riesgo tan altas, puesto que son los futuros profesionales de la salud que atenderán a diversos pacientes durante su práctica médica, así como la baja predisposición a participar de estudios en línea(25).

En conclusión, las prácticas de riesgos más frecuentes fueron la baja utilización de condón durante la última relación sexual, la utilización de alcohol durante la relación sexual y la baja realización de prueba para VIH. Se deberían comparar los resultados con los de estudios similares de la región y en los casos en los que hubiese diferencias, tratar de explicarlas.

\section{REFERENCIAS BIBLIOGRÁFICAS}

1. Delgado R. Características virológicas del VIH. Enferm Infecc Microbiol Clin. 2011; 29(1):58-65.

2. Organización Mundial de la Salud (OMS). DIEZ DATOS SOBRE EL VIH/SIDA. OMS. [Consultado: 01 de enero de 2017]: Disponible http://www.who.int/features/factfiles/hiv/e s/

3. Antón FA, Espada JP. Consumo de sustancias y conductas sexuales de riesgo para la transmisión del VIH en una muestra de estudiantes universitarios. Anales de psicología. 2009;25:344-50.

4. Frentz $D$, van de Vijver $D$, Abecasis $A$, Albert J, Hamouda O, Jørgensen L, et al. Patterns of transmitted HIV drug resistance in Europe vary by risk group. PloS one 2015; 9(4), e94495.

5. Moure-Rodríguez L, Doallo $\mathrm{S}$, Juansalvadores $\mathrm{P}$, Cadaveira $\mathrm{F}$, Caama $\mathrm{F}$. Consumo intensivo de alcohol y cannabis, y prácticas sexuales de riesgo en estudiantes universitarios. Gac Sanit 2016;30(6):43843.

6. Leoni AF, Martelloto GI, Jakob E, Cohen JE, Aranega CI. Conductas sexuales y riesgo de infecciones de transmisión sexual en estudiantes de medicina de la universidad nacional de córdoba. DST-J bras Doenças Sex Transm 2005;17(2):93-8.

7. Ruiz-Sternberg AM, Beltrán-Rodríguez J, Latorre-santos C, Ruiz-Sternberg JE. ¿Existe asociación entre los comportamientos de riesgo en salud sexual y reproductiva y el ser estudiante de medicina? Estudio de corte transversal. Bogotá (Colombia). Revista Colombiana de Obstetricia y Ginecología 2010;61(2):1218.

8. Arguello $M$, Bautista $Y$, Carvajal J, De Castro K, Diaz D, Escobar M, et al. Estilos de vida en estudiantes del área de la salud de Bucaramanga. Revista de psicología Universidad de Antioquia 2009;1(2) 27-41.

9. Campo Y, Pombo L, Teheran A. Estilos de vida saludable y conductas de riesgo en estudiantes de medicina. Revista de la Universidad Industrial de Santander. Salud 2016; 48(3):301-9. 
10. Departamento de Salud del Estado de Nueva York. Cuestionario sobre riesgo de enfermedades de transmisión sexual/ Virus de Inmunodeficiencia Humana/Hepatitis; [Consultado en enero de 2017]. Disponible en: https://www.health.ny.gov/forms/doh4336es.pdf

11. Ríos-Gonzalez CM. Prácticas de riesgo para transmisión de VIH en adultos de la ciudad de General Elizardo Aquino, Diciembre 2014 - Enero 2015. Paraguay. Horiz Med. 2015;15(2): 12-8.

12. Thai TT, Jones MK, Harris LM, Heard RC. The association between symptoms of mental disorders and health risk behaviours in Vietnamese HIV positive outpatients: a cross-sectional study. BMC Public Health 2017; 17(1): 250.

13. Rius C, Binefa G, Casabona J. Epidemiologia de la infección por el VIH/sida y su relación con otras infecciones de transmisión sexual Perspectiva de futuro. Enferm Infecc Microbiol Clin 2004; 22(7):419-29.

14. Plames $M$, Gómez $A B$, Gras $M E$, FontMayolas S. Cambios en las percepciones de riesgo frente al SIDA de los estudiantes universitarios durante la última década. C Med Psicosom 2005/2006; 76/7:39-45.

15. García Alúm NE, Valdés Vento $A C$, Gutiérrez Machado AJ, Forcelledo Llano CR, Delgado Rodríguez A. Evolución de los conocimientos para la prevención del VIH/SIDA en estudiantes de medicina. Rev Ciencias Médicas [Internet]. 2009;13 (3): 19-29.

16. Mazzitelli M, Caridá G, Scigliano C, Concetta Vallone $\mathrm{M}$, et al. Knowledge of HIV infection and transmission: a knowledge, attitudes, beliefs and practices (KABP) survey among a simple of studentes at the "Magna Graecia", University of Catanzaro. Ann Ist Super Sanitá 2016; 53(4): 530-5.

17. Vera Lina $M$, López $N$, Ariza N, Díaz L. Asociación entre el área de estudio y los conocimientos y comportamientos frente a la transmisión del VIH/SIDA en los estudiantes de la Universidad Industrial de Santander. Colomb Med. 2004;35: 62-8.

18. De Benedictis-Serrano GA, Rios-González CM. The LGBT community and HIV: An incorrect medical judgment. Travel Med Infect Dis. 2018 May 1. pii: S14778939(18)30090-5.

doi: 10.1016/j.tmaid.2018.04.017.

19. Van-der Hofstadt CJ, Antón-Ruiz FA, Tirado $S$, Navarro-Cremades F. Hábitos sexuales en mujeres estudiantes de medicina. C. Med. Psicosom 2007/2008; 84/85: 32-48.

20. García Alúm NE, Méndez Romero G, Gutiérrez Machado AJ, Pérez Labrador O, Aranda RR. Actitudes hacia el uso del condón masculino para la prevención del $\mathrm{VIH} /$ sida en estudiantes de medicina. Rev Ciencias Médicas [Internet]. 2011; 15 (3):38-49. Disponible en: http://scielo.sld.cu/scielo.php?script=sci_a rttext\&pid $=$ S156131942011000300005\&lng=es.

21. Cortés Alfaro A, García Roche R, Ochoa Soto R. Comportamiento sexual y uso del condón en estudiantes de la facultad de tecnología de la salud. Rev Cubana Med Trop [Internet].2015 67(2): 202-12. Disponible en: http://scielo.sld.cu/scielo.php?script=sci_a rttext\&pid $=$ S0375$07602015000200007 \&$ Ing $=$ es.

22. Vergara $K$, Cárdenas $S$, Martínez $F$. Consumo de alcohol y problemas asociados en estudiantes de una universidad pública de Cartagena. Rev Colomb Psiquiat 2011; 40(2): 215-28.

23. Quimbayo-Díaz JH, Olivella-Fernández MC. Consumo de marihuana en estudiantes de una universidad colombiana. Rev salud pública 2013;15 (1): 32-43.

24. Rios-González CM, Flores-Enríquez JF. Reflections on the epidemiology of HIV/AIDS in Latin America. Journal of infection and public health.2018; 11 (3): 449-50.

25. Ríos-González CM. Importancia de la oferta de tamizaje del VIH en Paraguay. CIMEL [revista en internet]. 2017; 22 (2).

26. Ríos-González CM, D Benedictis GA. Predisposición para la participación de estudiantes de medicina en encuestas online: una reflexión sobre la experiencia. FEM (Ed. impresa).2017; 20 (4): 205. 